\title{
Universal Power Law in the Orientational Relaxation in Thermotropic Liquid Crystals
}

\author{
Dwaipayan Chakrabarti, Prasanth P. Jose, Suman Chakrabarty, and Biman Bagchi* \\ Solid State and Structural Chemistry Unit, Indian Institute of Science, Bangalore 560012, India
}

(Received 7 July 2005; published 3 November 2005)

\begin{abstract}
We observe a surprisingly general power law decay at short to intermediate times in orientational relaxation in a variety of model systems (both calamitic and discotic, and also lattice) for thermotropic liquid crystals. As all these systems transit across the isotropic-nematic phase boundary, two power law relaxation regimes, separated by a plateau, emerge, giving rise to a steplike feature (well known in glassy liquids) in the single-particle second-rank orientational time correlation function. In contrast to its probable dynamical origin in supercooled liquids, we show that the power law here can originate from the thermodynamic fluctuations of the orientational order parameter, driven by the rapid growth in the second-rank orientational correlation length.
\end{abstract}

DOI: 10.1103/PhysRevLett.95.197801

PACS numbers: 61.20.Lc, 64.70.Md, 64.70.Pf

Thermotropic liquid crystals are known to exhibit interesting, often exotic, dynamical properties which are the subject of great fundamental interest [1,2]. For calamitic liquid crystals that comprise rodlike molecules, the approach to increasingly ordered mesophases on lowering temperature involves an isotropic to nematic (I-N) transition and then a nematic to smectic-A transition that are both believed to be only weakly first order with considerable characteristics of continuous transitions [1-6]. On the other hand, discotic liquid crystals that consist of disclike molecules exhibit a transition from an isotropic to a nematic-discotic phase and onward to a columnar phase rather than a smectic phase $[1,2]$.

Surprisingly, dynamics across the I-N phase transition appears to have been investigated mostly at low frequencies or long times (milliseconds to nanoseconds) [1]. However, optical Kerr effect (OKE) measurements [7] by Fayer et al. have recently revealed a power law relaxation near the I-N phase boundary as well as in the nematic phase of rodlike molecules having aspect ratios between 3 and 4 $[8,9]$. While the power law decay appears at short (a few picoseconds) to intermediate (between tens of picoseconds and a few nanoseconds) times in the isotropic phase near the I-N transition [8], multiple power laws persist even at long times in the nematic phase [9]. Although a few theoretical analyses exist in the literature $[8,10]$, the origin and the scope of this dominant power law relaxation are poorly understood at present.

In order to understand this power law decay, we have carried out a long and extensive molecular dynamics (MD) simulation study of orientational relaxation in a variety of model systems for thermotropic liquid crystals, both continuous and lattice. The continuous systems in our study consist of ellipsoids of revolution. We have used the GayBerne pair potential [11], which is an elegant generalization of the extensively used isotropic Lennard-Jones potential, to explicitly incorporate anisotropy in both the attractive and the repulsive parts of the interaction with a single-site representation for each ellipsoid of revolution
[12]. For a representative calamitic system, we have considered a system of 576 prolate ellipsoids of revolution with an aspect ratio of 3 that is comparable to those of the rodlike molecules recently studied experimentally by Fayer and co-workers. We have investigated the system along an isotherm at several densities.

In the quest of a universal power law behavior, if at all there is any, we have in addition undertaken simulations of a system of 500 oblate ellipsoids of revolution with an aspect ratio of 0.345 . We have used in this case a GayBerne pair potential that has been modified for disclike molecules by Bates and Luckhurst [13]. The discotic system has been studied here along an isobar at several temperatures.

The well known Lebwohl-Lasher (LL) model is a prototype of lattice models [14], where the particles are assumed to have uniaxial symmetry and are represented by three-dimensional spins, located at the sites of a simple cubic lattice, interacting through a pair potential of the form $U_{i j}=-\epsilon_{i j} P_{2}\left(\cos \theta_{i j}\right)$. Here $\epsilon_{i j}$ is a positive constant $\epsilon$ for nearest neighbor spins $i$ and $j$ and zero otherwise, $P_{2}$ is the second-rank Legendre polynomial, and $\theta_{i j}$ is the angle between the spins $i$ and $j$. In this work, we have also considered a 1000-particle LL lattice system to study pure orientational dynamics across the I-N transition. The simplicity of the model allows us to study a larger system size. In our MD simulations, the system undergoes a transition from the isotropic to nematic phase at the temperature $T \simeq 1.14$.

In Figs. 1(a)-1(c), we show in log-log plots the time evolution of the single-particle, second-rank orientational time correlation function (OTCF) observed for the calamitic, discotic, and lattice systems, respectively, across the I$\mathrm{N}$ phase transition [15]. The I-N transition is marked by a jump in the orientational order parameter $S$, defined for an $N$-particle system as the largest eigenvalue of the ordering matrix $Q: Q_{\alpha \beta}=\frac{1}{N} \sum_{i=1}^{N} \frac{1}{2}\left(3 e_{i \alpha} e_{i \beta}-\delta_{\alpha \beta}\right)$, where $e_{i \alpha}$ is the $\alpha$ component of the unit orientation vector $\hat{\mathbf{e}}_{i}$ along the principal symmetry axis of the $i$ th ellipsoid of revolution in 

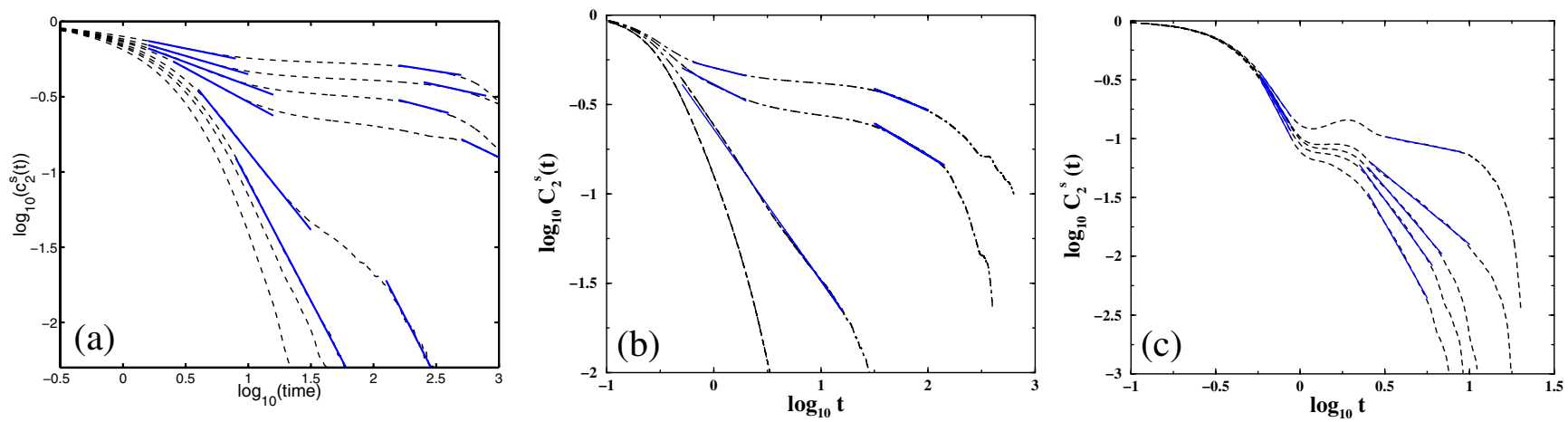

FIG. 1 (color online). Time evolution of the single-particle second-rank OTCF in log-log plots for the (a) calamitic system, (b) discotic system, and (c) lattice system. The black dashed lines are simulation data corresponding to increasing order parameter from bottom to top. The blue solid lines show the fits to the data over the time regimes where the decay follows a power law. (a) Along an isotherm at $T=1.0$ at several densities: $\rho=0.285,0.295,0.3,0.305,0.31,0.315,0.32$, and 0.33 . The I-N transition density is bracketed by $\rho=0.305$ and $\rho=0.315$ with $\rho=0.31$ falling on the transition region. (b) Along an isobar at $P=25.0$ at several temperatures: $T=2.991,2.693,2.646$, and 2.594. The I-N transition temperature is bracketed by $T=2.693$ and $T=2.646$. (c) At several temperatures: $T=1.213,1.176,1.160,1.149,1.134$.

the space fixed frame [16]. The single-particle second-rank OTCF $C_{2}^{s}(t)$ is defined by

$$
C_{2}^{s}(t)=\frac{\left\langle\sum_{i} P_{2}\left(\hat{\mathbf{e}}_{i}(0) \cdot \hat{\mathbf{e}}_{i}(t)\right)\right\rangle}{\left\langle\sum_{i} P_{2}\left(\hat{\mathbf{e}}_{i}(0) \cdot \hat{\mathbf{e}}_{i}(0)\right)\right\rangle}
$$

where the angular brackets stand for equilibrium ensemble averaging. Note in these figures the emergence of the power law decay at short to intermediate times near the I-N phase boundary. Figures 1(a)-1(c) further show that, as the systems transit across the I-N phase boundary, two power law relaxation regimes, separated by a plateau, appear, giving rise to a steplike feature. The intriguing feature is the universality in qualitative behavior, given that widely different model systems are under consideration. Although the values of the power law exponents vary considerably from one system to another, certain trends are apparent. The power law exponent values decrease monotonically for all the systems on approaching the I-N phase boundary from the isotropic side and undergo a rather sharp drop to a value below 0.45 on crossing the boundary. The only exception to this is the early power law exponent for the LL lattice system, where the two power law relaxation regimes are evident even in the isotropic phase with a rather weak temperature sensitivity for the early power law exponent. In this case, a hump appears in the plateau region on approaching the I-N phase boundary. This can be attributed to the inertial effects because of low damping of the orientational motion. Near the I-N phase boundary where the continuous systems show a single power law relaxation regime, the exponent has a value above (1.582) or below (0.848) unity, depending upon whether system is calamitic or discotic. The values of the exponents in these systems seem to rule out grouping them together in a common universality class. The weakly first order nature of the I$\mathrm{N}$ transition also precludes the existence of such strict universality. Note that the overall relaxation behavior looks surprisingly similar to the decay of a self-intermediate scattering function in supercooled liquids where such power laws are well known [17].

In experiments, one can probe orientational relaxation through the decay of the OKE signal, which is given by the negative of the time derivative of the collective secondrank OTCF $C_{2}^{c}(t)$ [7-9]. The latter, which is defined by

$$
C_{2}^{c}(t)=\frac{\left\langle\sum_{i} \sum_{j} P_{2}\left(\hat{\mathbf{e}}_{i}(0) \cdot \hat{\mathbf{e}}_{j}(t)\right)\right\rangle}{\left\langle\sum_{i} \sum_{j} P_{2}\left(\hat{\mathbf{e}}_{i}(0) \cdot \hat{\mathbf{e}}_{j}(0)\right)\right\rangle},
$$

is computationally demanding, particularly at long times. In order to set a direct link with experimental results, we show the temporal behavior of the OKE signal in the loglog plots in the respective systems across the I-N phase transition in Figs. 2(a)-2(c). The short-to-intermediatetime power law regime is evident in the OKE signal for all three systems. While a power law decay of the OKE signal has been recently observed experimentally in calamitic systems near the I-N phase boundary and in the nematic phase $[8,9,18]$, our prediction for the discotic systems could be tested in experiments.

We now address the origin of this general power law relaxation with two plausible explanations. The first originates from the observation that such power laws are well known in supercooled liquids where an elegant expression is provided by the mode coupling theory (MCT) $[19,20]$. In the MCT description [19], a nonlinear dependence of the memory function (the longitudinal viscosity) on the density-density time correlation function and the feedback between the viscosity and the dynamic structure factor lead to the power law relaxation, which is, therefore, purely dynamical in origin. Since the memory function involved here pertains to orientational motion, as a first approximation, we may replace it by the rotational friction, which can be obtained from the evaluation of the torque-torque time correlation function by using the time dependent density 

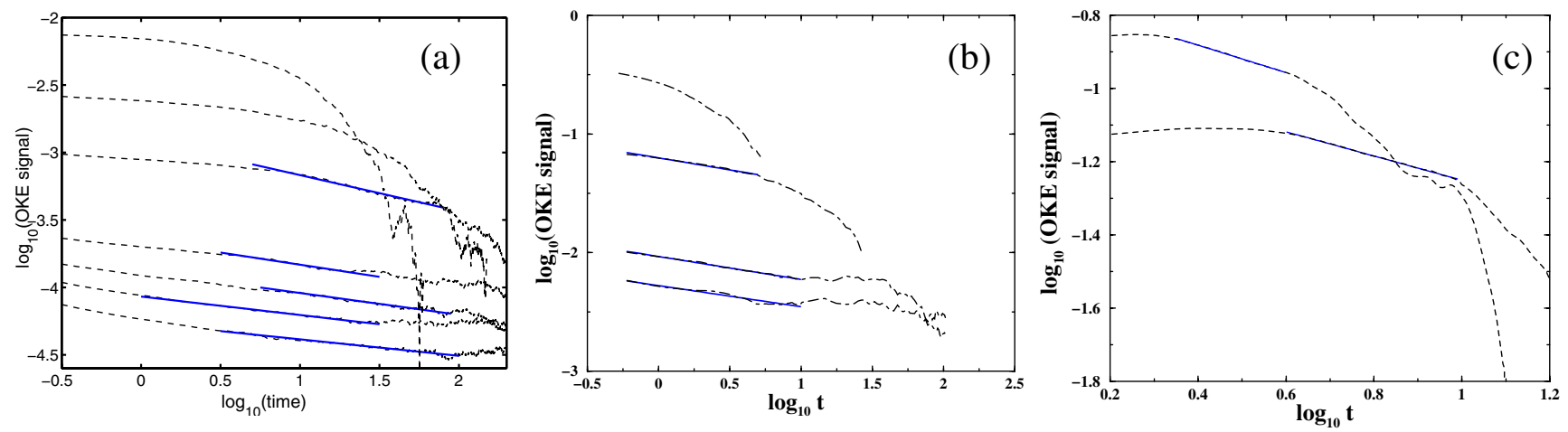

FIG. 2 (color online). Time evolution of the collective second-rank OTCF at short to intermediate times in log-log plots for the (a) calamitic system, (b) discotic system, and (c) lattice system. The black dashed lines are simulation data corresponding to increasing order parameter from bottom to top. For all three cases, the blue solid lines show the fits to the data over the time regimes, if any, where the decay follows a power law: $t^{-\alpha}$. The values of the power law exponent $\alpha$ are given below in the parentheses. (a) Along an isotherm at $T=1.0$ at several densities: $\rho=0.285, \rho=0.3, \rho=0.305(\alpha=0.26), \rho=0.31(\alpha=0.18), \rho=0.315(\alpha=0.16), \rho=0.32$ ( $\alpha=0.13$ ), and $\rho=0.33$ ( $\alpha=0.12)$. (b) Along an isobar at $P=25.0$ at several temperatures: $T=2.991, T=2.693(\alpha=0.208)$, $T=2.646(\alpha=0.194)$, and $T=2.594(\alpha=0.178)$. (c) At two temperatures: $T=1.176(\alpha=0.374)$ and $T=1.149(\alpha=0.33)$.

functional theory. In this approach, the wave number $(k)$ and the orientation dependent fluctuating density of the mesogen is the natural slow variable which can be expanded in spherical harmonics. The following expression for the singular part of the memory function can then be obtained for axially symmetric mesogens:

$$
\Gamma^{\operatorname{Sing}}(z)=\frac{3 k_{B} T \rho}{8 \pi^{3} I} \int_{0}^{\infty} d k k^{2} \sum_{l, m} c_{l, l, m}^{2}(k) S_{l, m}(k, z),
$$

where $c_{l, l, m}(k)$ is the $(l, l, m)$ component of the spherical harmonic expansion of the two particle direct correlation function, $S_{l, m}(k, z)$ is the same for the $(l, m)$ component of the orientational dynamic structure factor, and $z$ is the Laplace frequency [21,22]. Near the I-N transition, dynamic structure factor $S_{20}(k, z)$ slows down dramatically due to the rapid and diverging growth of $S_{20}(k)$ in the long wavelength limit [23]. It can be shown that this leads to a power law behavior of the memory function: $\Gamma^{\operatorname{Sing}}(z)=$ $A^{\prime} z^{-1 / 2}, A^{\prime}$ being a constant, at short to intermediate times. Use of this memory function in the Mori-Zwanzig continued fraction representation of the dynamic structure factor gives $S_{20}(t)=\exp \left(a^{2} t\right) \operatorname{erfc}\left(a t^{1 / 2}\right)$, where $a$ is a constant. This has the required power law behavior. Thus, here the power law arises due to diverging correlation length, unlike in supercooled liquids, where no such divergence of correlation length is involved. Near the I-N phase boundary, on the other hand, the free energy surface can play a direct role in the short time power law. Near the I-N transition, an equation of motion for the fluctuating orientational order parameter $(\delta S)$, a nonconserved variable [24], can be written as follows:

$$
\frac{d \delta S(t)}{d t}=-\int d t^{\prime} \Gamma\left(t-t^{\prime}\right) \frac{\delta F}{\delta(\delta S)}\left(t^{\prime}\right)+R(t),
$$

where $\Gamma$ is a damping coefficient, $F(\delta S)$ is the Landau- de Gennes free energy as a function of the orientational order parameter, and $R(t)$ is a random velocity term related to $\Gamma$ by the fluctuation-dissipation theorem. As the temperature approaches the critical temperature $T_{c}$, the free energy surface becomes soft. If one uses the Landau free energy expansion $\delta F=A(T)(\delta S)^{2}+B(T)(\delta S)^{3}+$ $C(T)(\delta S)^{4}$, then it can be shown that Eq. (4) can also give rise to a power law decay of $\Delta \bar{S}(t) / \Delta \bar{S}(0)$ at short to intermediate times, where the bar denotes the nonequilibrium ensemble average, without the necessity of invoking power law behavior of the memory function. Note that both explanations involve diverging second-rank orientational correlation length $\xi_{2}(T)$, which is in turn attributed to the softening of the coefficient $A(T)$ in the Landau-de Gennes theory because $A(T) \propto \xi_{2}(T)^{-2}$. However, in Eq. (4) higher order terms in the free energy expansion are important at short to intermediate times.

In order to quantitatively understand the role of order parameter fluctuations, we have computed the distribution of the fluctuating orientational order parameter $S$. In Fig. 3, we show this distribution at four temperatures across the I-N phase boundary along an isochor. For the finite size effect, the average orientational order parameter has nonzero finite value (of the order of $N^{-1 / 2}$ ) even in the isotropic phase. While the distribution of $S$ is unimodal in both the isotropic and nematic phases, it becomes bimodal near the I-N phase boundary with two peaks at $S_{i}$ and $S_{n}$ $\left(S_{i}<S_{n}\right)$ corresponding to an isotropiclike and a nematiclike configuration, respectively. The dominant peak shifts from $S_{i}$ to $S_{n}$, and then the distribution becomes unimodal as the system settles into the nematic phase. In the same figure, we have also shown the order parameter dependence of the free energy obtained from the distribution. Such a bimodal distribution could be observed only because of the weakly first order nature of the I-N transition (that is, the requirement of nucleation is not stringent). 

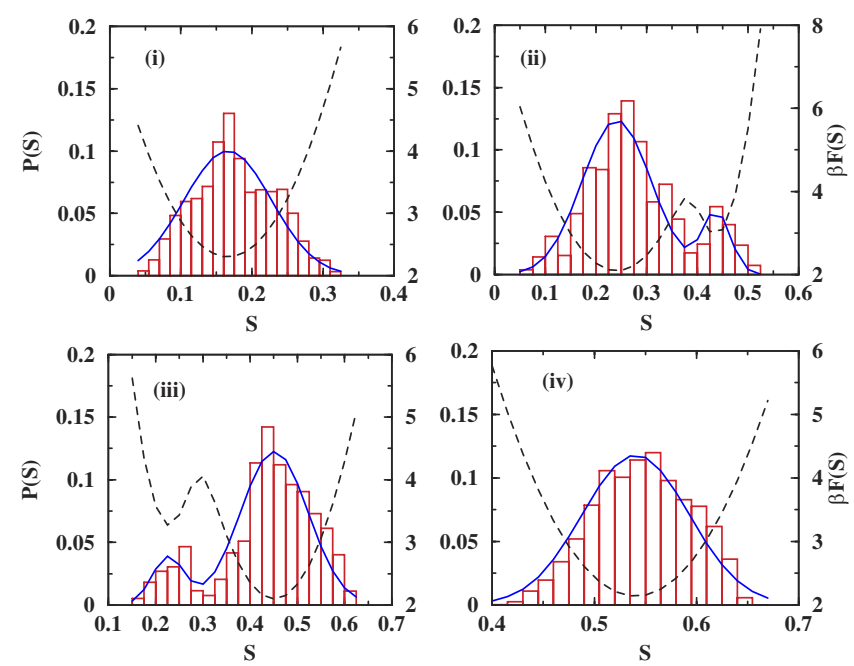

FIG. 3 (color online). The distribution $P(S)$ of the orientational order parameter $S$ for a 256 particle calamitic system at four state points across the I-N transition along an isochor at the density $\rho=0.32$ : (i) $T=1.495(\langle S\rangle=0.176$ ), (ii) $T=1.390$ ( $\langle S\rangle=0.280)$, (iii) $T=1.324(\langle S\rangle=0.435)$, and (iv) $T=1.191$ $(\langle S\rangle=0.547)$. The blue solid lines are fits to the histograms (red solid lines) with a Gaussian function for (i) and (iv) and with a linear combination of two Gaussian functions for (ii) and (iii). The black dashed line is the scaled free energy $F$, where $\beta=$ $1 /\left(k_{B} T\right)$, obtained from the fit function $P(S)$ through $\beta F(S)=$ $-\ln (P(S))$.

Note that the free energy barrier between isotropic and nematic phases is only of the order of $0.01 k_{B} T$ per particle. Resulting large fluctuations in $S$ can give rise to a power law decay of $\Delta \bar{S}(t) / \Delta \bar{S}(0)$ as discussed above.

If the power law decay is driven by the growth of the orientational correlation length, then its duration is expected to increase with the aspect ratio of the mesogens. We have, therefore, carried out a study of rodlike mesogens with aspect ratio 3.8 and confirmed this expectation.

The emergence of the power law relaxation in all three systems under consideration here near the I-N transition is due to the rapid growth of the orientational correlation length, which is also responsible for the universal features. Unlike in glassy systems, the power law decay may reflect large thermodynamic fluctuations of the order parameters, as shown in Fig. 3. Finally, our prediction of power law decay for discotic liquid crystals could be tested in experiments.

We thank S. Ramaswamy and C. Dasgupta for helpful discussions. This work was supported by the grant from DST, India. D. C. acknowledges UGC, India for providing financial support.
*Electronic address: bbagchi@sscu.iisc.ernet.in

[1] P. G. de Gennes and J. Prost, The Physics of Liquid Crystals (Clarendon, Oxford, 1993).

[2] S. Chandrasekhar, Liquid Crystals (Cambridge University Press, Cambridge, England, 1992).

[3] W. L. McMillan, Phys. Rev. A 4, 1238 (1971).

[4] P. G. de Gennes, Solid State Commun. 10, 753 (1972).

[5] B. I. Halperin, T. C. Lubensky, and S.-K. Ma, Phys. Rev. Lett. 32, 292 (1974).

[6] A. Yethiraj and J. Bechhoefer, Phys. Rev. Lett. 84, 3642 (2000).

[7] Y.-X. Yan and K. A. Nelson, J. Chem. Phys. 87, 6240 (1987); 87, 6257 (1987).

[8] S.D. Gottke, H. Cang, B. Bagchi, and M.D. Fayer, J. Chem. Phys. 116, 6339 (2002).

[9] J. Li, I. Wang, and M.D. Fayer, J. Phys. Chem. B 109, 6514 (2005).

[10] P. P. Jose and B. Bagchi, J. Chem. Phys. 120, 11256 (2004).

[11] J. G. Gay and B. J. Berne, J. Chem. Phys. 74, 3316 (1981).

[12] J. T. Brown, M. P. Allen, E. M. del Río, and E. de Miguel, Phys. Rev. E 57, 6685 (1998).

[13] M.A. Bates and G. R. Luckhurst, J. Chem. Phys. 104, 6696 (1996).

[14] P. A. Lebwohl and G. Lasher, Phys. Rev. A 6, 426 (1972).

[15] All the quantities reported here for prolate and oblate ellipsoids of revolution are given in reduced units defined in terms of the Gay-Berne potential parameters $\epsilon_{0}$ and $\sigma_{0}$ : length in units of $\sigma_{0}$, temperature in units of $\epsilon_{0} / k_{B}, k_{B}$ being the Boltzmann constant, and time in units of $\left(\sigma_{0}^{2} m / \epsilon_{0}\right)^{1 / 2}, m$ being the mass of the ellipsoids of revolution. We set the mass as well as the moment of inertia of each of the ellipsoids of revolution equal to unity. In the case of the lattice system, temperature is scaled by $\epsilon / k_{B}$. The moment of inertia of each particle has been set equal to unity.

[16] C. Zannoni, in Advances in the Computer Simulations of Liquid Crystals, edited by P. Pasini and C. Zannoni (Kluwer Academic, Dordrecht, 2000).

[17] W. Kob, J. Phys. Condens. Matter 11, R85 (1999).

[18] H. Cang, J. Li, V. N. Novikov, and M. D. Fayer, J. Chem. Phys. 118, 9303 (2003).

[19] U. Bengtzelins, W. Götze, and A. Sjölander, J. Phys. C 17, 5915 (1984).

[20] W. Götze and M. Sperl, Phys. Rev. Lett. 92, 105701 (2004).

[21] C. G. Gray and K. E. Gubbins, Theory of Molecular Fluids (Clarendon, Oxford, 1984), Vol. 1.

[22] B. Bagchi and A. Chandra, Adv. Chem. Phys. 80, 1 (1991).

[23] M.P. Allen and M. A. Warren, Phys. Rev. Lett. 78, 1291 (1997).

[24] P. C. Hohenberg and B. I. Halperin, Rev. Mod. Phys. 49, 435 (1977). 